\title{
Pois et tourteau de colza : deux matières premières complémentaires pour I'alimentation animale
}

\author{
Corinne PEYRONNET ${ }^{1}$ \\ Frédéric PRESSENDA ${ }^{2}$ \\ Laurent ALIBERT ${ }^{3}$ \\ Isabelle BOUVAREL ${ }^{4}$ \\ 1 ONIDOL, \\ 12, avenue George V, \\ 75008 Paris \\ <c.peyronnet@prolea.com> \\ 2 CEREOPA, \\ 16 rue Claude Bernard, \\ 75231 Paris cedex 05 \\ 3 IFIP Institut du Porc, \\ 34 boulevard de la Gare, \\ 31500 Toulouse \\ 4 ITAVI-UMT BIRD, \\ 37380 Nouzilly
}

Pour ses élevages, l'Europe importe plus de $70 \%$ de ses besoins en protéines qui sont essentiellement satisfaits par le tourteau de soja représentant à lui seul $64 \%$ de la consommation européenne de protéines (sur la campagne 2008-2009).

En France, la dépendance protéique est moindre puisque le déficit en matières riches en protéines (MRP) est de $53 \%$, le tourteau de soja couvrant $59 \%$ des besoins. Ce sont les tourteaux d'oléagineux métropolitains colza et tournesol qui sont les deuxième et troisième fournisseurs de protéines assurant respectivement 22 et $7 \%$ de la couverture.

Le développement de la production de biodiesel en France, issu en majorité d'huile de colza, a largement contribué au développement de la culture de colza et par conséquent à la production et la consommation de tourteau de colza. Ainsi, la consommation de tourteau de colza a plus que doublé depuis la campagne 2003-2004 atteignant près de 2,3 millions de tonnes en 2009.

Les protéagineux et tout particulièrement le pois, sont des sources de protéines intéressantes pour l'alimentation animale mais qui ne couvraient plus qu'1,5\% des besoins en 2008-2009 contre plus de $14 \%$ en 1993 au maximum de la production française.

\begin{abstract}
Pea and rapeseed meal are protein rich feedstuffs. Despite their differences regarding composition their amino acid profiles are quite complementary. For growing and finishing pigs it is easy to formulate diets with pea and rapeseed meal all together without any soybean meal. Prices of feed are not increased. For poultry diets, peas and rapeseed meal can replace soybean meal in diets for slow growing birds but price of feed increases. Prices ratio of pea and wheat and rapeseed and soybean meals have to decrease to make pea and rapeseed meal more competitive for poultry nutrition.
\end{abstract}

Key words: pea, rapeseed meal, feedstuffs, pigs, poultry
Même si les tourteaux d'oléagineux métropolitains et les protéagineux ont connu des développements inverses avec un ciseau de leur contribution au bilan MRP en 1995, ces matières premières ne sont pas pour autant concurrentes. Elles peuvent en effet s'avérer complémentaires vis-à-vis de la dépendance protéique de la France.

\section{Des débouchés historiquement différents}

De par leur composition nutritionnelle spécifique (figure 1), le pois et le tourteau de colza trouvent classiquement des valorisations distinctes vis-à-vis des différentes espèces animales. En effet, alors que le pois a toujours été très

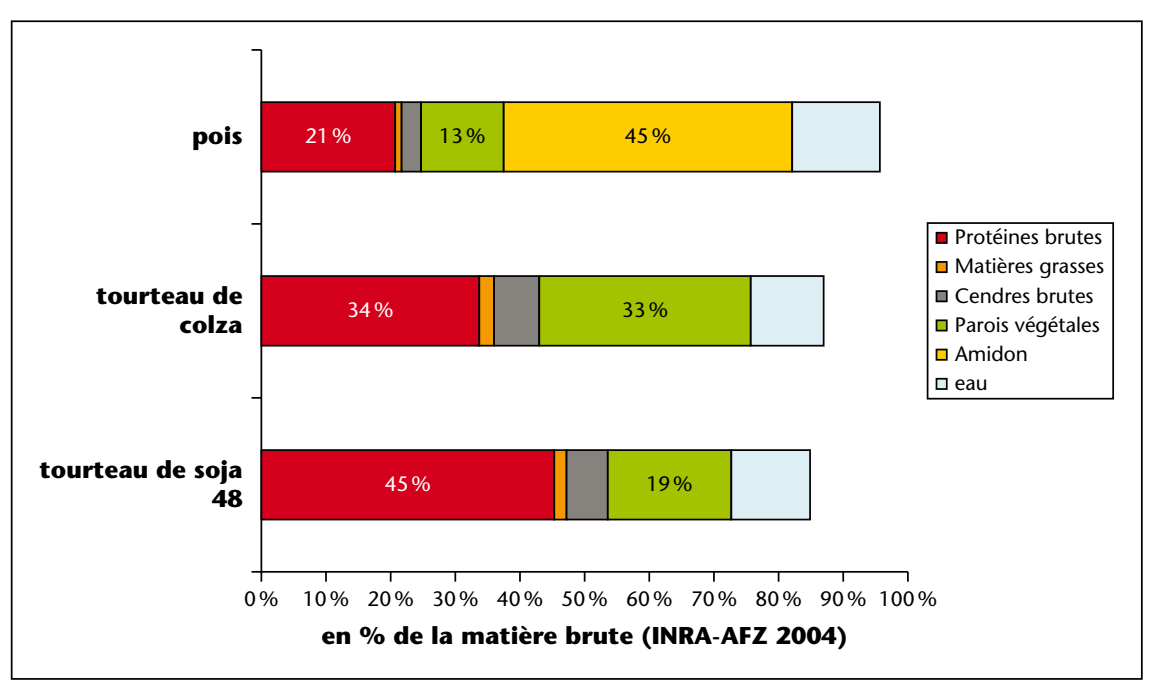

Figure 1. Composition du pois et des tourteaux de colza et de soja. 
majoritairement incorporé dans les formules pour porcs, le tourteau de colza est encore principalement utilisé (à $71 \%$ ) par les ruminants avec $46 \%$ pour les vaches laitières et $25 \%$ pour les bovins de type viande selon une estimation du modèle Prospective Aliments du CEREOPA qui simule, à l'échelle de la France entière, la consommation des différentes matières premières destinées au secteur des aliments composés industriels. Ce même modèle indique que le deuxième débouché est constitué par les formules pour porcs (24\%) alors que les volailles, fortes consommatrices de tourteau de soja nécessaire à la couverture des besoins élevés en protéines et énergie, n'en utiliseraient que $3 \%$. On peut cependant penser que ce débouché pourrait s'élargir, notamment au travers des formules pour volailles à croissance plus lente et en particulier dans les formules de production sous Signes Officiels de Qualité recherchant des sources de protéines moins concentrées et d'origine locale ou encore dans les formules pour poules pondeuses sous réserve que les poules soient insensibles à la sinapine présente dans le colza.

Il semble que, même si la croissance de la consommation de tourteau de colza doit se faire par une progression de son incorporation dans les formules pour porcs et volailles, l'arrivée de quantités significatives de protéagineux sur le marché ne devrait pas être un frein à leur développement.

\section{Pois et tourteau de colza : des sources de protéines différentes et complémentaires sur le plan nutritionnel}

Pois et tourteau de colza sont des MRP bien différentes. Le pois ne contient que $21 \%$ de protéines comparativement aux tourteaux de colza et de soja qui en contiennent 34 et $45 \%$ de la matière brute respectivement (figure 1) mais sa richesse en amidon lui confère une valeur énergétique élevée pour les monogastriques comme l'indique la figure 2 .

Ainsi, il apparaît que le pois présente la valeur énergétique volaille (Energie Métabolisable Apparente à bilan azoté nul : EMAn) la plus élevée avec $2490 \mathrm{Kcal}$ par kg de matière brute selon les tables INRA-AFZ de 2004 soit $9 \%$ au-dessus de celle du tourteau de soja. La forte teneur en fibres du tourteau de colza pénalise sa valeur énergétique qui se trouve très nettement inférieure avec $1460 \mathrm{Kcal} \mathrm{par} \mathrm{kg.}$

En porc, l'expression de la valeur énergétique en valeur Energie Nette (EN) pour la croissance est encore plus favorable au pois qui présente une valeur de $2320 \mathrm{kcal} / \mathrm{kg}$ de matière brute, soit près de 20 \% supérieure à celle du tourteau de soja.

Le mélange de pois et tourteau de colza permet donc de s'approcher de la valeur énergétique du tourteau de soja auquel il peut donc se substituer.

La complémentarité des pois et tourteau de colza peut également s'apprécier par les teneurs en Acides Aminés (AA) indispensables (figure 3).

Alors que le pois présente la protéine la plus riche en lysine $(7,3 \%$ contre 6,1 et 5,3\% respectivement pour le tourteau de soja et le tourteau de colza), le tourteau de colza présente lui les plus fortes concentrations en AA soufrés méthionine et cystine $(4,5 \%$ contre 2,9 et $2,3 \%$ respectivement pour tourteau de soja et pois).
L'association du pois et du tourteau de colza permet ainsi de s'approcher du profil en AA du tourteau de soja. II convient par contre de noter que le pois est plus pauvre en tryptophane que les 2 tourteaux et qu'il est donc parfois nécessaire de complémenter en tryptophane pour obtenir un apport équilibré en AA.

\section{Pois et tourteau de colza en formules porcs}

L'IFIP Institut du porc a réalisé de nombreux essais d'incorporation du tourteau de colza et/ou du pois en alimentation porcine. Ainsi, les limites maximales d'incorporation recommandées sont indiquées dans le tableau 1.

En formulation, il est tout à fait possible d'incorporer pois et tourteau de colza en formules porcelets $2^{\mathrm{e}}$ âge et porcs en croissance et finition

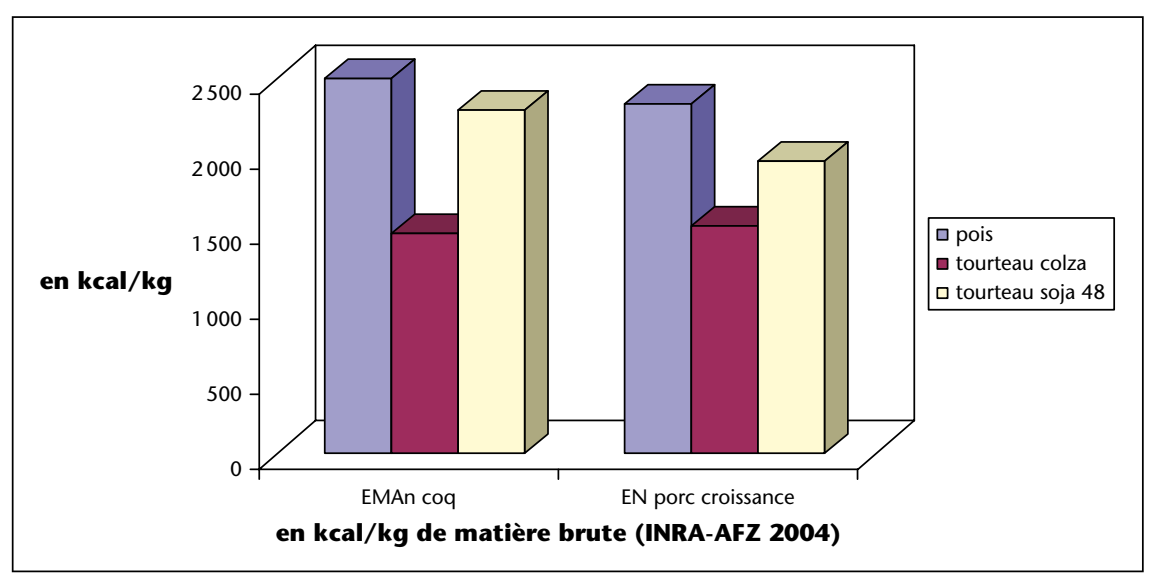

Figure 2. Valeurs énergétiques pour porcs et volailles du pois et des tourteaux de soja et de colza.

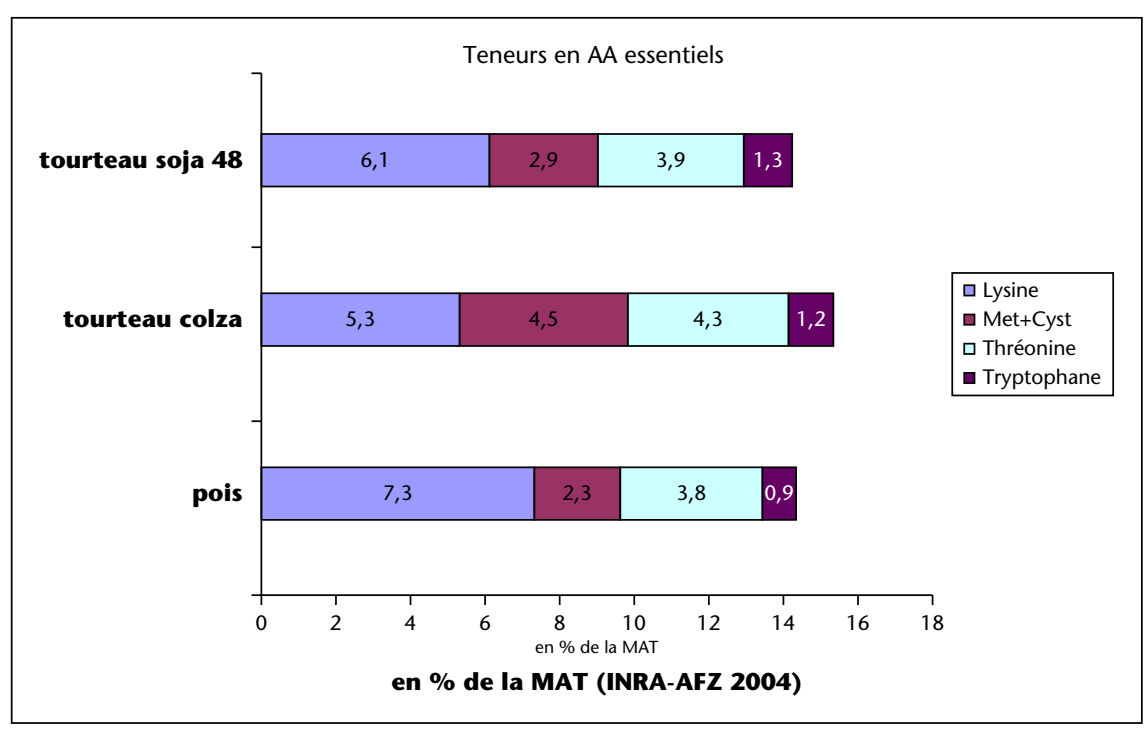

Figure 3. Complémentarité des pois et tourteau de colza sur le plan des teneurs en Acides Aminés (AA) indispensables. 
Tableau 1. Limites maximales d'incorporation des pois et tourteaux de colza en alimentation porcine en $\%$.

\begin{tabular}{|l|l|l|l|}
\hline & Porcelet $\mathbf{2}^{\mathbf{e}}$ âge & $\begin{array}{l}\text { Porc croissance } \\
\text { et finition }\end{array}$ & $\begin{array}{l}\text { Truie gestante } \\
\text { et allaitante }\end{array}$ \\
\hline Pois & 30 & $\mathrm{NL}$ & $\mathrm{NL}$ \\
\hline Tourteau de colza & 12 & 15 & 10 \\
\hline
\end{tabular}

$\mathrm{NL}$ : non limité.

Source : table d'alimentation pour les porcs IFIP édition 2002.

tout en respectant les normes Corpen sur la teneur en matières azotées totales (MAT) et le phosphore digestible. C'est ce que montre I'IFIP dans le tableau 2 qui présente une formulation réalisée sur une conjoncture de décembre 2009 et qui compare des régimes classiques avec tourteau de soja à des régimes incorporant pois et tourteau de colza.

Même si l'incorporation de pois et tourteau de colza simultanément ne permet pas dans la formule pour porcelet $2^{\mathrm{e}}$ âge de se passer de tourteau de soja, celle-ci permet d'incorporer des quantités significatives de pois et tourteau de colza (25 et $12 \%$ respectivement) tout en réduisant le coût de la formule de façon significative (de $7 \%$ rapporté au prix des $10 \mathrm{MJ}$ $\left.d^{\prime} E N\right)$. Les taux d'incorporation de tourteau de colza butent alors sur les niveaux maximums conseillés du fait d'un besoin élevé en protéi- nes. L'amélioration importante (7\%) du prix de la formule peut s'expliquer par un ratio de prix favorable entre tourteau de colza et tourteau de soja (0.56). Celui-ci, constaté en décembre 2009, est en effet plus favorable que la valeur moyenne du ratio qui s'établit sur la période 2001 à juillet 2010 à 0.62 comme l'indique la figure 4.

A contrario, cette conjoncture de décembre 2009 présente un prix du pois élevé avec un différentiel de prix par rapport au blé de $59 €$ par tonne ce qui est important comparativement à I'historique des prix observés depuis 1994 et présentés dans la figure 5 qui fait apparaître un écart moyen de $28 €$ par tonne depuis plus de 15 ans.

Il convient cependant de noter que toutes les formules obtenues sont très légèrement moins concentrées en EN. Cette réduction de la concentration énergétique des rations des porcs est possible du fait de la capacité qu'a le porc d'ajuster sa consommation moyenne journalière $(\mathrm{CM})$ à la concentration énergétique du régime sans conséquence sur le gain moyen quotidien (GMQ), I'indice énergétique et la qualité de carcasse.

Ces travaux de formulation d'aliments pour porcs montrent qu'il est tout à fait possible de diversifier l'approvisionnement en protéines par l'apport de protéines locales sans pénaliser les performances techniques et économiques.

\section{Pois et tourteau de colza en formules volailles}

Un exercice similaire à celui réalisé sur les porcs peut être fait sur des formules pour volailles telles que le poulet label (les volailles standard à croissance rapide ne peuvent quasi pas se passer de tourteau de soja nécessaire à la formulation d'aliments suffisamment concentrés pour répondre à leurs besoins).

Dans le même contexte de décembre 2009, le CEREOPA a comparé des régimes classiques avec tourteau de soja à des régimes avec pois et tourteau de colza en imposant des taux d'incorporation significatifs (tableau 3).

La conjoncture de prix de décembre 2009 ne permet pas d'incorporer naturellement pois et

Tableau 2. Comparaison de formules avec tourteau de soja et l'association tourteau de colza et pois (IFIP).

\begin{tabular}{|c|c|c|c|c|c|c|}
\hline \multirow{2}{*}{\begin{tabular}{|l} 
MP en \% \\
blé
\end{tabular}} & \multicolumn{2}{|c|}{ Porcelet $2^{\mathrm{e}}$ âge } & \multicolumn{2}{|c|}{ Porc croissance } & \multicolumn{2}{|c|}{ Porc finition } \\
\hline & 59 & 40,1 & 82,5 & 61,9 & 85,1 & 66 \\
\hline orge & 15 & 14,7 & 0 & 0 & 0 & 0 \\
\hline tourteau soja & 21,6 & 4,2 & 14,3 & 0 & 11,9 & 0 \\
\hline tourteau colza & 0 & 12 & 0 & 9,5 & 0 & 6 \\
\hline pois & 0 & 24,6 & 0 & 25,4 & 0 & 25 \\
\hline $\mathrm{AM}+\mathrm{AA}$ en $\%$ & 4,4 & 4,4 & 3,2 & 3,2 & 3 & 3 \\
\hline EN (MJd'EN/kg) & 9,5 & 9,3 & 9,9 & 9,7 & 10 & 9,8 \\
\hline Prix de 10 MJ EN (soja = 100) & 100 & 93 & 100 & 97 & 100 & 97 \\
\hline Prix en $€ / t($ soja $=100)$ & 100 & 91 & 100 & 94 & 100 & 96 \\
\hline \multicolumn{7}{|l|}{ AM : aliment minéral } \\
\hline \multicolumn{7}{|c|}{$\begin{array}{l}\text { conjoncture décembre } 2009 \text { en } € / \text { tonne } \\
\text { (parité Rennes) }\end{array}$} \\
\hline blé & 123 & & & & & \\
\hline orge & 106 & & & & & \\
\hline tourteau de soja & 328 & & & & & \\
\hline tourteau de colza & 185 & & & & & \\
\hline pois & 182 & & & & & \\
\hline
\end{tabular}




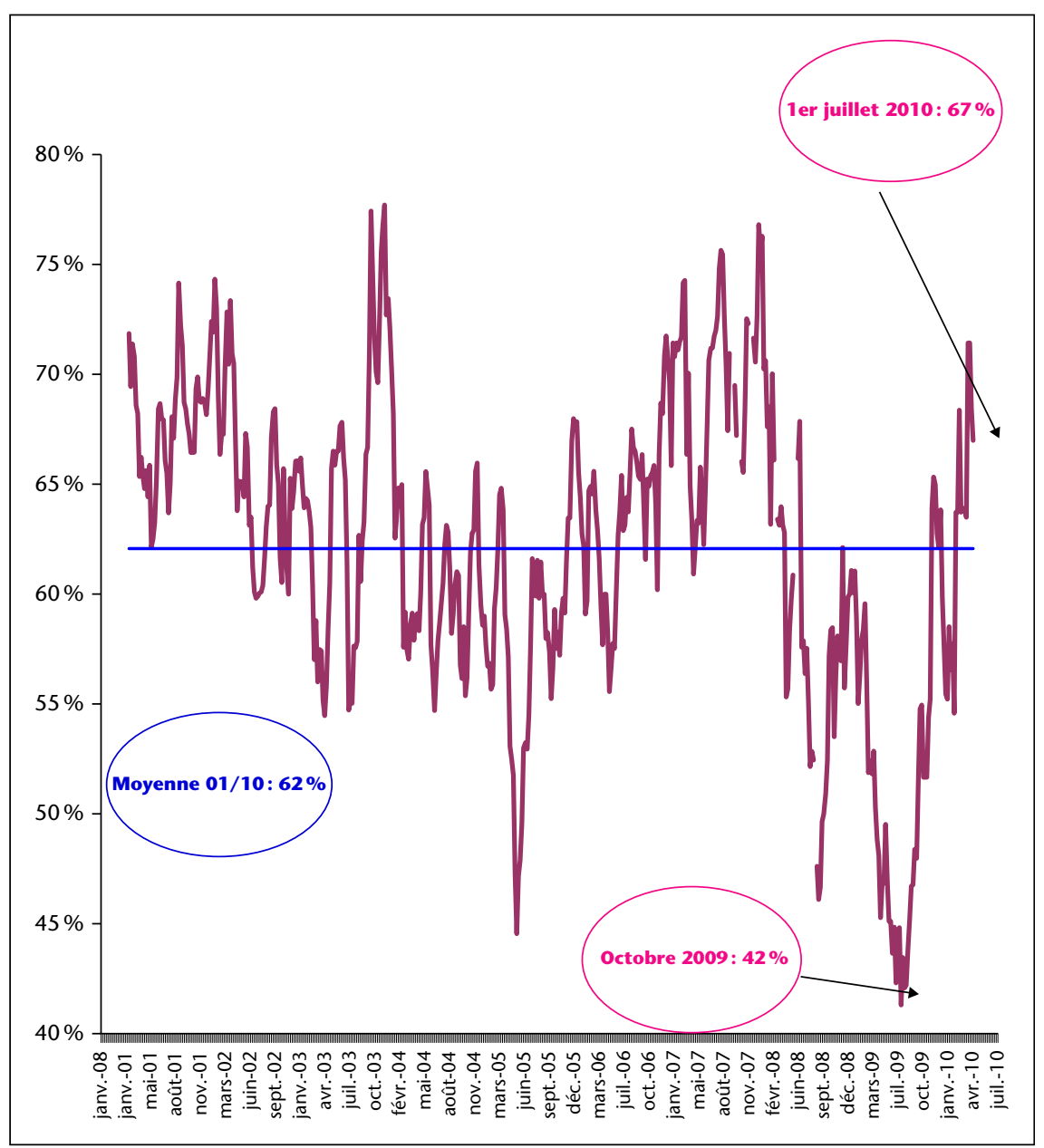

Figure 4. Rapport PRIX TT COLZA/TT SOJA (TT colza Rouen/TT soja Lorient).

tourteau de colza car leur prix d'intérêt respectif est inférieur aux prix de marché. Si I'on force I'incorporation du pois à $15 \%$ et du tourteau de colza à $10 \%$ en les intégrant à leur prix d'intérêt (soit - $13 \%$ pour le pois et - $6 \%$ pour le tourteau de colza par rapport aux prix de marché) la formule obtenue présente alors un surcoût de 3,2 \% comparativement à la formule avec tourteau de soja conventionnel. Cette hausse de prix est cependant comparable à celle obtenue en formulant avec un tourteau de soja non OGM pour lequel le surcoût s'établit à 2,9 \%.

Ces résultats mettent en évidence la moindre adaptation des MRP locales pois et tourteau de colza aux formules volailles qu'aux formules porcs dans lesquelles elles sont incorporées plus facilement sans entraîner de surcoût de formule.

Il apparaît que les rapports de prix entre pois et blé et entre tourteau de colza et tourteau de soja respectivement de 1,31 et 0,53 sont encore trop élevés et pénalisants pour le prix d'efforts de prix (par rapport aux prix actuels) à consentir sur les matières premières que sont les pois et tourteau de colza.

\section{Effet de la valeur nutritionnelle du tourteau de colza sur son intérêt en formules volailles}

Indépendamment de l'étude de l'association du pois et du tourteau de colza en formules volailles se pose la question de l'effet de la valeur nutritionnelle du tourteau de colza sur son intérêt économique. Un état des lieux de la valeur nutritionnelle des tourteaux de colza produits dans les usines de trituration françaises a ainsi été réalisé en 2009 à l'INRA de Nouzilly. II apparaît que la valeur EMA mesurée sur coq varie de 1570 à $1800 \mathrm{kcal} / \mathrm{kg}$ de matière brute et que la valeur moyenne obtenue est de $1650 \mathrm{kcal}$ comparativement à la valeur de 1460 indiquée dans les tables INRA-AFZ de 2004. De tels écarts de valeur énergétique sont déterminants sur le prix d'intérêt calculé dans différentes formules pour volailles. En effet, le tableau 4 indique, pour 4 types de formules pour volailles sur une période allant de juillet 2007 à novembre 2009, les écarts de prix d'intérêt entre les différents tourteaux de colza (max, moyen et tables) et comparativement au tourteau de soja. Lorsque ces écarts sont exprimés en ratio de prix d'intérêt il apparaît qu'alors que le tourteau de colza des tables peut être intéressant jusqu'à un ratio de prix de $64 \%$ par rapport au tourteau de soja, le tourteau de colza présentant la meilleure valeur énergétique peut être intéressant lorsque son prix atteint jusqu'à $71 \%$ du prix du tourteau de soja (et ce, dans des formules pour poulet certifié croissance). Ce rapport de prix est bien moindre dans les formules destinées aux animaux à croissance plus forte comme les poulets standard.

Sur la base de la seule amélioration de la valeur énergétique des tourteaux de colza testés dans cette étude, il ressort donc une amélioration du prix d'intérêt de cette matière première dans les différentes formules volailles. En rapportant ce prix d'intérêt au prix de marché du tourteau de soja, on note une amélioration du ratio prix d'intérêt du tourteau de colza/prix du tourteau de soja qui varie de 11 à $27 \%$ pour le colza max et de 6 à $15 \%$ pour le colza moyen selon les formules.

\section{Conclusion}

L'étude de l'intérêt nutritionnel et économique de l'incorporation de MRP locales dans les régimes des porcs et volailles met en évidence la faisabilité technique de l'association du pois et du tourteau de colza dans ces formules. L'inté- 


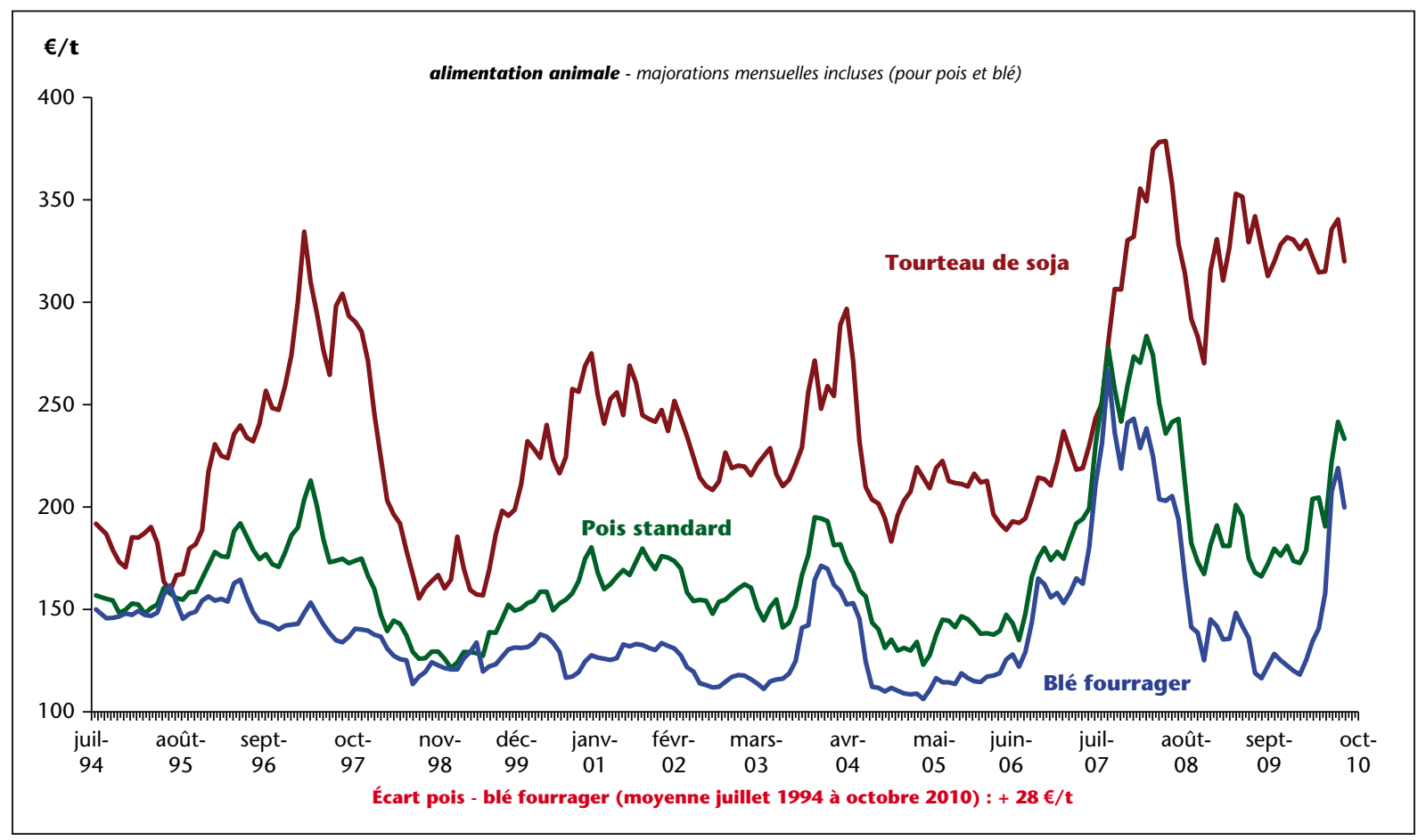

Figure 5. Prix rendus Centre Bretagne.

Tableau 3. Comparaison de formules volailles label avec tourteau de soja ou association de pois + tourteau de colza (CEREOPA).

\begin{tabular}{|c|c|c|c|c|}
\hline \multirow{2}{*}{\begin{tabular}{|l} 
Matières premières \\
Taux d'incorporation en \%
\end{tabular}} & \multicolumn{3}{|l|}{ Formules } & \\
\hline & Tourteau de soja & T. colza et pois (au PI) & T. soja non OGM & \\
\hline blé & 82,7 & 64,2 & 43,5 & \\
\hline maïs & & & 34,4 & \\
\hline tourteau de soja & 13,6 & & & \\
\hline t. soja non OGM & & & 13,6 & \\
\hline tourteau de colza & & 10 & & \\
\hline pois & & 15 & & \\
\hline autres & 0,2 & 6,9 & 5 & \\
\hline $\mathrm{AM}+\mathrm{AA}$ & 3,6 & 3,9 & 3,5 & \\
\hline Prix formule en indice & 100 & 103,2 & 102,9 & \\
\hline Conjoncture décembre 2009 : & Prix rendu Bretagne & pois/blé ou T.colza/T.soja & Prix d'intérêt (PI) & pois/blé ou T.colza/T.soja \\
\hline blé & 122 & & & \\
\hline maïs & 133 & & & \\
\hline pois & 184 & 1,51 & 160 & 1,31 \\
\hline tourteau soja & 327 & & & \\
\hline tourteau soja non OGM & 362 & & & \\
\hline tourteau colza & 185 & 0,57 & 173 & 0,53 \\
\hline prix exprimés en $€ /$ tonne & & & & \\
\hline
\end{tabular}


Table 4. Écarts de prix d'intérêt et de ratio de prix tourteau de colza/tourteau de soja dans différentes formules pour volailles

CEREOPA, 2010 (entre juillet 2007 et novembre 2009)

\begin{tabular}{|c|c|c|c|c|}
\hline & $\begin{array}{l}\text { Poulet } \\
\text { crois. } \\
\text { Stand. }\end{array}$ & $\begin{array}{l}\text { Poulet } \\
\text { fin. } \\
\text { Stand. }\end{array}$ & $\begin{array}{l}\text { Poulet } \\
\text { certifié } \\
\text { crois. }\end{array}$ & pondeuse \\
\hline \multicolumn{5}{|l|}{ ÉCART DE PRIX D'INTÉRÊT (€/t) } \\
\hline colza max - tables & 39 & 36 & 21 & 29 \\
\hline colza moy - tables & 22 & 20 & 11 & 16 \\
\hline soja - colza maximum & 142 & 136 & 95 & 111 \\
\hline \multicolumn{5}{|l|}{ RATIO DE PRIX D'INTÉRÊT } \\
\hline colza tables/soja & 0,44 & 0,46 & 0,64 & 0,57 \\
\hline colza moyen/soja & 0,5 & 0,53 & 0,68 & 0,62 \\
\hline colza max/soja & 0,56 & 0,58 & 0,71 & 0,66 \\
\hline \multicolumn{5}{|l|}{ ÉVOLUTION DU RATIO DE PRIX D'INTÉRÊT } \\
\hline colza moyen/soja par rapport à colza tabes/soja & $14 \%$ & $15 \%$ & $6 \%$ & $6 \%$ \\
\hline colza max/soja par rapport à colza tables/soja & $27 \%$ & $26 \%$ & $11 \%$ & $16 \%$ \\
\hline
\end{tabular}

rêt économique de ces associations est très dépendant des conjonctures économiques et surtout des types de rations concernées. L'association pois et tourteau de colza semblent tout à fait adaptée à I'alimentation des porcs. Par contre en ce qui concerne les volailles, il apparaît que la valeur énergétique plutôt faible du tourteau de colza soit un réel frein à son incorporation et que celui-ci soit contraint de voir son prix baisser fortement pour être incorporé dans les formules pour volailles. II semble donc plus que jamais nécessaire d'améliorer les connaissances sur la valeur réelle des matières premières et en particulier du tourteau de colza à destination des volailles. II est également nécessaire d'engager des travaux visant à améliorer la valeur nutritionnelle des tourteaux en augmentant la valeur énergétique et la digestibilité des différents constituants. Des solutions sont certainement à rechercher dans les procédés technologiques et enzymatiques ou encore dans la sélection végétale de façon à réduire les effets négatifs des fractions pariétales.

Enfin, la compétitivité des MRP métropolitaines dans I'alimentation des animaux monogastriques dépend de façon plus générale des prix de l'énergie et des protéines présentes sur le marché de l'alimentation animale, celles-ci étant susceptibles de varier largement en qualité et en quantité.

\section{RÉFÉRENCES}

IFIP, ADAESO, ARVALIS, CETIOM, UNIP. Tables d'alimentation pour les porcs, édition 2002.

Sauvant D, Perez JM, Tran G (coord.). Tables de composition et de valeur nutritive des matières premières destinées aux animaux d'élevage, AFZ-INRA, $2^{\mathrm{e}}$ édition, 2004.

Vigour B. Etude de la digestibilité du tourteau de colza chez la volaille et optimisation des formules alimentaires, mémoire d'ingénieur PURPAN, INRA, ONIDOL, mai 2010.

Pressenda F. Note provisoire sur l'intérêt de différents tourteaux de colza dans les formules volailles, CEREOPA, avril 2010. 